\title{
Di-NONAME-LIB: Logging only Dirty Chunks for Efficient Management of Dynamic Memory Based Optimistic Simulation Objects
}

\begin{abstract}
A recent work has presented the design and implementation of a software library, named NONAME-LIB (name removed for blind review) supporting transparent log/restore facilities for optimistic simulation objects with generic memory layout. This library offers the possibility to allocate/deallocate memory chunks within the object state via standard API, and performs log/restore of the object state via pack/unpack techniques, exploiting ad-hoc meta-data concisely identifying the object state layout at each point in simulation time. In this paper we complement such a library with a software architecture offering the following additional advantages: (i) run-time identification of chunk updates within the dynamic memory map, (ii) reduced checkpoint latency and increased effectiveness in memory usage thanks to log/restore facilities based on (periodic) snapshots of the whole simulation object state, taken via the incremental copy of the modified (dirty) chunks only. Hence, Di-NONAME-LIB (Dirty-NONAME-LIB). Our approach is based on software instrumentation techniques (suited for LINUX and the ELF format), targeting memory update references performed by the application level software, and on a lightweight run-time monitoring mechanism providing minimal overhead while tracking the exact memory addresses and the size of memory areas dirtied by the execution of each event. Also, Di-NONAME-LIB has been designed for portability across 32-bits and 64-bits Intel compliant architectures, thus covering a wide spectrum of offthe-shelf machines. Some performance results for an evaluation of effectiveness and scalability of our proposal (vs the state size) are provided.
\end{abstract}

\section{Introduction}

State management is one of the most critical aspects in optimistic simulation systems, which involves both performance and transparency aspects. In particular, log/restore mechanisms for optimistic simulation objects' states should likely be lightweight (in terms of both latency and memory usage) and act with no explicit intervention or awareness by the application programmer (e.g. in terms of direct interfacing between the application level software and the state $\log$ /restore sub-system).

A recent work [19] has presented a memory management library (NONAME-LIB), implemented in C technology, which offers completely transparent log/restore facilities while supporting, at the same time, a general programming model where the state of a simulation object can be scattered on dynamically allocated memory chunks. In particular, NONAME-LIB, has been designed for integration within traditional-style optimistic simulation kernels (see, e.g., $[9,3,13,19])$, and allows the application programmer to employ standard malloc services within event handler modules (e.g. for changing the current layout of the object state).

In this work we complement NONAME-LIB via a software architecture which adds new capabilities within the memory management subsystem, while maintaining the same transparency level. Specifically, we present the design and implementation of software modules that are able to track state updates (with arbitrary granularity) occurring during event processing. These modules also offer $\log /$ restore facilities based on (periodic) snapshots of the whole state of the simulation object built by incrementally copying only dirty chunks within the dynamic memory map associated with the state layout. Hence the name DiNONAME-LIB (Dirty-NONAME-LIB) for the out-coming memory management system.

On the side of state logging, the present proposal can provide direct performance advantages over the original NONAME-LIB due to the reduced amount of memory copy operations. In fact, a complete snapshot of the simulation object state involves logging a subset of the chunks currently belonging to the memory map (those that have been dirtied since the last checkpoint was taken). It can also provide indirect advantages (e.g. via increased locality) thanks to the reduction of the memory usage for storing the snapshots.

On the other hand, the new architecture involves runtime monitoring activities to determine the exact addresses that are referenced in memory update operations by the application level software. To efficiently support this task, we have designed and implemented a lightweight memory write tracking mechanism. It is based on transparent application software instrumentation, and is tailored for LINUX and standard ELF objects/executables on both 32-bits and 64-bits Intel compliant hardware architectures (namely IA32 and X86-64 architectures).

From a methodological point of view, the literature work closest to the present proposal is [27], where a transparent incremental state saving method is supported via automatic editing of the application executable. However, the substantial differences between our software architecture and the capabilities of the state management system in [27] are that we cope with dynamic memory and support recoverability for each permitted operation (allocation, deallocation and 


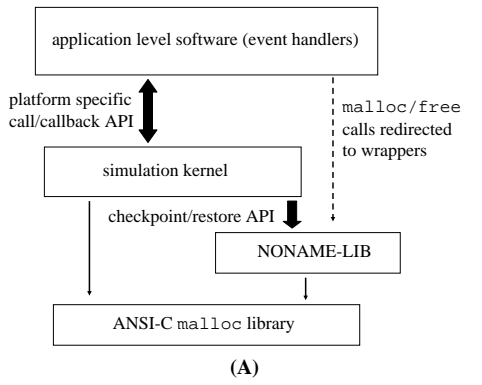

(A)

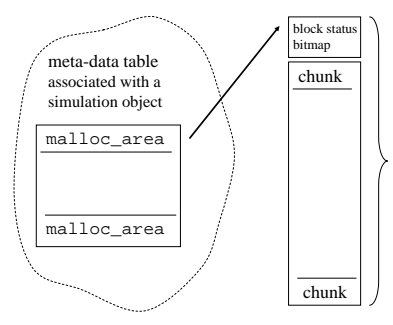

(B)
Figure 1. Software Architecture and $(A)$ main NONAME-LIB data structures (B).

update), which are not provided in that work.

The remainder of this paper is structured as follows. In Section 2 we provide an overview of NONAME-LIB as the basis for the comprehension of the whole software system. In Section 3 the innovative capabilities and the design of the associated modules are presented. Related work is discussed in Section 4. Experimental data for the assessment of the effectiveness of Di-NONAME-LIB are presented in Section 5.

\section{Overview of NONAME-LIB}

From an architectural point of view, NONAME-LIB can be seen as a wrapper of ANSI-C malloc/free services, which is transparently interposed, via simple compile/linking time directives, between the application level code and the traditional malloc library (a schematization of this approach is shown in Figure 1.A). NONAME-LIB also offers an API for integration with the simulation kernel, which consists of a set of services supporting memory management operations specifically oriented to $\log /$ restore activities proper of the optimistic synchronization scheme.

For each simulation object hosted by the simulation kernel, NONAME-LIB maintains a meta-data table of malloc_area entries. Each entry keeps information about a block of contiguous memory chunks (e.g. the memory location of the block), possibly allocated for serving memory requests for that object, and different entries are used for managing chunks of different sizes. As soon as a malloc request occurs for a given chunk size, the corresponding block is allocated by NONAME-LIB (via real malloc services) so that a contiguous number of those chunks is in practice pre-allocated for serving future requests with that same size (if any). This allows exalting memory contiguity for the state layout of each simulation object, which can favor performance during both event processing and $\log /$ restore operations. In addition, pre-allocation of contiguous chunks allows NONAME-LIB to use very concise meta-data for the identification of the status of each chunk (busy or free) within a block. In particular, a simple bitmap of so called "status bits" is used for the identification of those pre-allocated chunks that have also been delivered (and are currently in use) to the application software. To further optimize memory usage, NONAMELIB adopts a layout where the bitmap of status bits is col- located at the head of the pre-allocated block of chunks, and gets allocated only in case of real allocation of the corresponding block (see Figure 1.B for a schematization of the relation between the main data structures describing the simulation object memory map). Actually, the table of malloc_area entries can be expanded in case its entries have been saturated, and the simulation object goes on requesting more chunks during event processing activities.

Log/restore operations are supported by NONAME-LIB via pack/unpack techniques. For a log operation, the currently in use memory chunks are packed into a contiguous $\log$ buffer (which is dynamically allocated via the underlying malloc services), together with all the active malloc_area entries and the status bitmaps at the head of active blocks. For a restore operation, these data structures are extracted from the contiguous log buffer and put back in place. To make deallocation operations recoverable, each malloc_area entry also keeps information about the logical time (if any) at which the chunks inside a given block were all released. A block with no chunks currently allocated, and whose last chunk release occurred before the Global-Virtual-Time (GVT) can be deallocated via a free call towards the underlying malloc library. In such a case, the corresponding mal loc_area entry is set to non-active.

Actually, log operations exploit threshold-based optimizations, and an ad-hoc chunk allocation algorithm within each block, aimed at optimizing the trade-off between metadata management and memory copies of active chunks. In particular, each malloc_area keeps a counter identifying the percentage of in use chunks within the corresponding memory block. When the occupancy exceeds a given threshold, the memory block is entirely logged, without explicitly scanning the status bitmap in order to identify busy chunks. Also, chunk allocation within each memory block is similar to the LINUX algorithm for the selection of the next file descriptor to be assigned while opening an I/O channel. This algorithm keeps low block fragmentation and tends to have busy chunks clustered at the head of the memory block. Therefore, in case the bitmap needs to be scanned upon the log operation (i.e. when the block occupancy is under the threshold), the latency of the operation can benefit by the early stopping of the bitmap scan whenever all the in use chunks are really found clustered at the beginning of the memory block.

\section{Di-NONAME-LIB: Design and Implemen- tation}

As mentioned, Di-NONAME-LIB is based on new software modules able to track at run-time memory update references performed by the application software. It also uses data structures and modules which augment the capabilities of the original NONAME-LIB with the aim at tracking not only the current memory map of the simulation object (and its dynamic changes), but also the dirtying activities. This is done for increasing the efficiency of $\log /$ restore operations via the exploitation of an incremental approach.

In this section we first describe the techniques we have used for a lightweight instrumentation of the application level software, in order to support the memory update tracking process. Then we enter details of data structures' and 
modules' integration. Finally a discussion on the usage of third party libraries for application level software, and on how Di-NONAME-LIB handles memory dirtying operations occurring inside those libraries is provided.

\subsection{The Software Instrumentation Tool}

Software instrumentation has been realized via a software Parser/Modifier (PM) specifically designed for analyzing and rewriting ELF (Executable and Linking Format) objects generated by standard gcc compilers (versions 3 and 4) for IA-32 and X86-64 architectures. At the very base, PM works by parsing the object generated after linking together all the application level modules (third party libraries being excluded), and by identifying every memory write instruction inside this object, namely mov instructions with a memory location as the destination. The instrumentation process is then supported by PM via the insertion of a call instruction to an update_tracker module, edited in assembly language, which performs the identification of the exact memory address and the size (amount of bytes) involved in the memory update operations. Although this is a typical way for tracking memory update references (e.g. in the context of program debugging techniques [26]), the usage of this approach in optimistic simulation systems poses (more) stringent performance issues. In particular, the monitor should likely perform its job via very few machine instructions, in order not to significatively impact event execution latency.

To cope with such a performance target we have decided not to employ run-time disassembling of the memory reference instruction, which could be onerous (compared to the event execution latency of non-instrumented software) especially due to the complexity and variable format/length of the Intel instruction set. Instead, we have adopted an orthogonal technique where a software table associated with the update_tracker is built and populated during the compile-time instrumentation process. This table acts as a cache of disassembling results for memory write instructions.

In IA-32/X86-64 architectures, the address of each memory write operation depends on a set of up to four parameters, namely base, index, scale and displacement. The former two parameters correspond to register values (hence the parameters identify the registers containing the values), while the latter two correspond to specific values of fields inside the memory writing instruction. The instruction opcode tells which of those parameters are relevant. Also, the opcode, together with its prefixes, establish the real size of the memory area touched by the write operation. Hence, to cache the results of the disassemblig process, PM builds a table where each entry is structured as:

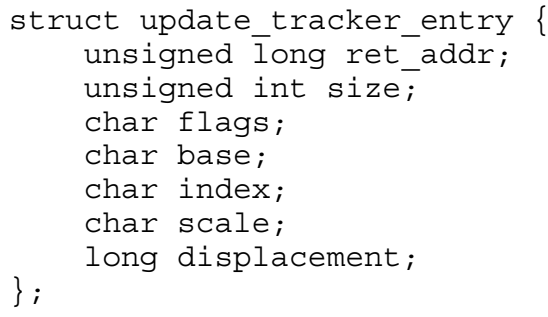

The flags field is used to identify which of the aforementioned four parameters (corresponding to subsequent fields inside this same data structure) are actually relevant and should be considered by the update_tracker for computing the exact address for the memory write operation. Also, the size field immediately indicates to the update_tracker the (compile-time defined) size of the memory area to be dirtied by the current memory write instruction $\left({ }^{1}\right)$. Finally, the ret_addr field indicates to the update_tracker where control will be returned after its execution. This field corresponds to the memory address of the write instruction which immediately follows the current instance of the call to the update_tracker. It has been inserted as a field of the update_tracker_entry data structure for allowing the disassembling results' table produced during the instrumentation process to be organized as a fast search hash-with-buckets table. (Recall that access to the update_tracker_entry associated with the memory write instruction occurs during the execution of the update_tracker. Hence it is a performance critical operation directly impacting the event execution cost for the instrumented version of the application software.) In particular, upon its activation, the update_tracker checks inside its own stack frame the value of the return address, which is used as the key for accessing the hash table maintaining update_tracker_entry records, and is compared to the ret_addr field inside these records for selecting the correct record within the bucket. Once this is done, the memory address for the write operation and the size of the memory being dirtied are easily computed by the monitor via a few machine instructions. Given that this computation can unpredictably change the value of the EFLAGS register on board of the CPU, this register value is saved by the update_tracker upon its activation together with general purpose ones, and is put back in place right before returning control to the memory write instruction for which the tracking process has been activated. Actually, PM can be parameterized in order to optimize the trade-off between the size of the hash-with-bucket table, and the access cost. Specifically, the instrumentation process can be asked to check whether the level of collision inside the hash table does not exceed a pre-specified value. In the negative case, PM can resize the hash-with-bucket table in order to reduce the actual bucket size. The potential drawback is the increase of unused table entries, while the benefit is the reduction of the update_tracker overhead when accessing the table. The most favorable case is when the bucket size is (or boils down to) 1, leading to $O(1)$ time complexity for the access to the hash table of update_tracker_entry records.

Actually, the update_tracker uses absolute addresses as keys for the hash table. In fact, as mentioned above, this module identifies the address of the memory write operation at run-time by accessing its own stack

\footnotetext{
${ }^{1}$ The only exception is for movs and stos instructions, specifically used for moving arbitrary size memory blocks. However, for those instructions, the information for identifying the destination address and the current size of the memory block being written is immediately available into predefined registers, namely EDI and ECX, which are directly accessible by the update_tracker.
} 
frame. In order for PM to be able to build the table of update_tracker_entry records via correct insertion of the absolute addresses of memory writing instructions inside the ret_addr field, we have exploited incremental linking facilities offered by standard linkers (e.g. Id on UNIX systems). In particular, the instrumentation process interacts with the linker for the definition of the exact (absolute) position of the object associated with application level software inside the executable layout. All the other modules (e.g. the simulation kernel object modules) will be then located by incrementally selecting absolute addresses compatible with the current position of the object associated with the application level software.

The insertion of the call to the update_tracker prior to the execution of a memory write instruction leads to a resize of the sections associated with the object file, and to the shift of instructions and other memory locations inside the object layout. Hence, PM also has to rewrite the headers associated with the ELF object, the relocation tables, and the off-sets used for the identification of memory addresses referenced by the software, e.g. the destination addresses for jmp instructions.

However, in IA-32/X86-64 processors not all destination addresses for $j \mathrm{mp}$ instructions that are found in the original software can be identified at compile time, and corrected via rewriting of the relocation tables. This occurs in case of so called register-jumps (also frequently referred to as indirect-branches), where the destination address is dynamically identified via the content of CPU registers. To cope with this issue, we have implemented a second run-time monitoring mechanism for supporting on-the-fly correction of destination addresses in register-jumps. Like in the aforementioned approach, this mechanism is based on the insertion of a call instruction to a second assembly level monitoring module, referred to as branch_corrector, prior to each register-jump in the original software. This monitoring module relies on a hash table similar to the previously described one, where each entry is associated with a single register-jump instruction, and keeps the information regarding which are the registers whose values determine the destination address for the jump operation. Also this table is built and populated at compile-time during the instrumentation process (again to avoid costly run-time disassembling techniques). Exploiting the information inside this table, branch_corrector evaluates the original destination address for the jump instruction (by reading the CPU registers that specify the destination value). Then it corrects this address on the basis of the amount of bytes by which the original destination was shifted inside the instrumented object layout (we recall that the shift is due to the insertion of the additional call instructions to the monitoring modules). To provide a lightweight mechanism for the address correction operation, PM generates a third table at compile-time, which is visible to branch_corrector. Each entry inside this table identifies an interval of addresses for which the instrumentation process gave rise to the same amount of shift inside the final (instrumented) memory layout. Such an offset is also maintained in each table entry. The table is ordered by interval extremes, and branch_corrector performs a logarithmic-cost binary search to retrieve the interval containing the original destination for the registerjump, and the offset to be applied for the correction. Such a correction cannot however be applied by modifying the values of the CPU registers involved in the jump instruction. This would otherwise result in an application inconsistent processor state. Instead, we have adopted a different approach where the original register-jump instructions (whose relevant information is anyway logged inside the hash table available to branch_corrector), are substituted at compile-time by PM with so called offset-jumps (not relying on CPU registers), where the destination address is maintained inside one field of the instruction, and is appropriately set by the on-the-fly correction mechanism. To support the rewrite operation of the appropriate instruction field at run-time, without impacting typical settings associated with memory protection, the offset-jump operation has been moved inside a run-time re-writable ELF section (adhoc created by exploiting compiler/linker facilities). Also, a jump-label instruction has been inserted in place of the offset-jump inside the original (non-rewritable) sections of the application code, which passes control to the offsetjump right after the brach_corrector module has rewritten the correct destination address (the offset) inside the ad-hoc re-writable section.

We note that efficient solutions for correcting registerjumps (e.g. via the avoidance of run-time disassembling) have practical relevance since register-jumps are typically generated by standard compilers (e.g. gCc version 3) for machine language translation of Switch-Case constructs. The constructs are relatively relevant in simulation applications (e.g. for flow control inside the event handler on the basis of the type of dispatched event), which supports the relevance of the optimizations we presented for limiting the cost of on-the-fly address correction.

Going back to instrumentation of memory write instructions and to the structure of the update_tracker module, we have further tailored the process of identifying memory locations that are dirtied during the execution of simulation events to the simulation object memory model originally offered by NONAME-LIB. In this model, memory locations associated with automatic variables (allocated inside the stack) do not belong to the object memory map, since they do not survive across different invocations of the event handler. Hence, all those memory write instructions that can be detected at compile-time to access the stack (e.g. mov instructions addressing memory via base pointer or stack pointer displacement) are not actually instrumented by PM. Anyway, in some cases write access into the stack cannot be recognized at compile time. For this reason, after having computed the address for the memory write operation, update_tracker compares it with the current value of the stack pointer. In case the access is an actual stack update, update_tracker simply returns (the memory update operation is of no interest for the management of the object memory map). Otherwise, the information about the identified memory address and the size of the area being dirtied is passed to the memory map manager whose structure is presented in the next section. 


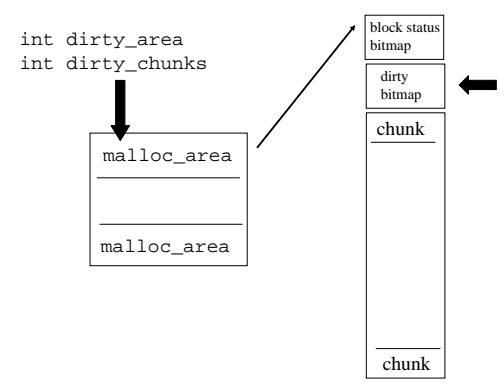

Figure 2. Main Memory Map Data Structures in Di-NONAME-LIB.

\subsection{Management of the Memory Map}

The original data structures and modules managing the simulation object memory map have been extended/modified in Di-NONAME-LIB in order to explicitly cope with the possibility to build complete state logs by incrementally logging only data that have been dirtied since the last log operation. To guarantee recoverability of each type of operation permitted on the memory map, namely chunk allocation/deallocation and chunk update, we need to deal with incremental log of both dirty data, namely dirty chunks, and dirty meta-data, namely dirty malloc_area entries associated with the memory map.

To track dirty chunks, a second bitmap, of so called dirty bits, has been associated with each block of preallocated chunks destined to a specific simulation object. As shown in Figure 2, this bitmap is located inside the same contiguous memory segment pointed by the corresponding malloc_area and containing the original status bitmap and the chunks destined for use by the overlying application in case of malloc requests. In terms of real storage, the dirty bitmap inherits the same features of the original status bitmap since its allocation occurs only in case the corresponding chunks gets really pre-allocated. Hence the extra storage occupancy for detecting chunks that have been dirtied since the last log operation well scales with the size of application destined storage. The bits inside the dirty bitmap are treated as sticky flags vs the memory write monitoring mechanism described in the previous section. Hence, a memory write operation performed by the application software can only result in a set operation of the dirty bit associated with the chunk being dirtied.

To track dirty meta-data we have added the following two integer fields inside the malloc_area data structure (see again Figure 2):

- dirty_area, which is used as a flag indicating whether any type of operation (allocation, deallocation or chunk dirtying) has occurred in the malloc_area since the last log.

- dirty_chunks, which explicitly counts the current number of in use chunks that have been dirtied in the malloc_area since the last log operation.
Once the memory map manager receives the address and the size of the memory area being dirtied from the memory_tracker, it identifies all the chunks that will be dirtied inside the memory map, and the associated malloc_area entries. Then the previous bitmaps and the dirty_chunks field are updated. Again in compliance with the original NONAME-LIB memory model, in case the address and the memory area being dirtied refer to locations outside the memory map of the currently executing simulation object (e.g. they refer to global variables outside the heap, for which recoverability is not provided in this memory model), the memory map manager simply returns control to the memory_tracker module. On the other hand, the dirty_area field inside the malloc_area is set to 1 each time a malloc/free call insisting on that area is performed by the application software.

\subsubsection{State Log Operations}

Via the exploitation of the additional fields inside each malloc_area, and of the dirty bitmaps, the logging activities performed by Di-NONAME-LIB have been differentiated in full-logs and incremental-logs. Both types of logs still result in packing the information to be logged inside a contiguous buffer allocated via the underlying malloc services. However, they pack different things (with consequently different costs). A full-log operation coincides with the original log supported by NONAME-LIB. Hence, the active malloc_area entries are packed inside the log buffer together with the in use chunks in the corresponding memory blocks, while the dirty bitmaps are not logged. The only additional tasks performed by the full-log operation in Di-NONAME-LIB is the reset of all the data structures explicitly used for tracking dirty data/meta-data (namely the dirty bitmaps and the two aforementioned fields inside the malloc_area). On the other hand, an incremental-log performs differentiated pack operations depending on the current value of those data structures. Specifically, for each active malloc_area entry we have the following cases:

A: dirty_area is set and dirty_chunks is zero. In this case the malloc_area is packed into the log buffer together with the status bitmap indicating the current allocation of chunks inside a given block. But the dirty bitmap and the currently in use chunks are not logged.

B: dirty_area is set and dirty_chunks is greater than zero. In this case the malloc_area is packed into the log buffer together with the status bitmap, the dirty bitmap and the chunks that are currently in use, which have been dirtied. All the other in use chunks are not logged.

$\mathrm{C}$ : dirty_area is not set. In this case, no information associated with the area is logged at all.

As for the case of full-logs, incremental-logging also involves the re-set of all the data structures tracking dirty data/meta-data. This occurs independently of the actual case among the aforementioned ones. 
We finally underline that incremental state log operations no way require to be forced at each simulation event, but can be taken periodically. In fact they are based on recognizing memory portions that have been dirtied since the last log, independently of the amount of events actually performing the dirtying operations. Hence, with Di-NOANMELIB state reconstruction at whichever simulation time can be supported via a mix of state restore (i.e. copy back) from the $\log$ (this procedure will be illustrated in the next section), and classical coasting forward.

\subsubsection{State Restore Operations}

Similarly to the original version of NONAME-LIB, each log is stamped with the current simulation time, and all the logs (full and incremental) are linked together as a chain. When a restore operation needs to be executed at simulation time $T$, the log chain is searched to determine the more recent log with time less than or equal to $T$ (logs with time greater than $T$ are simply discarded since they refer to causally inconsistent memory maps). In case the log found is a full one, then a restore operation is executed by simply unpacking all the logged data and putting them back in place (like in the original version of NONAME-LIB). A different restore algorithm is executed in case the log found is an incremental one. Specifically, the following steps are iterated by backward traversing the chain of logs:

1. A malloc_area found inside the log buffer, which has not been restored, is put back in place inside the meta-data table. The associated status bitmap is also copied back from the log buffer (recall that independently of the type of log and of the specific case for incremental-logging, a logged malloc_area is always associated with the corresponding status bitmap inside the $\log$ buffer to guarantee recoverability of chunk allocation/deallocation operations).

2. Each dirty chunk found inside the log and associated with the malloc_area, which has not yet been restored in a previous iteration while backward traversing the log, is copied back in its correct position inside the corresponding memory block.

The iterative restore procedure stops when all the active malloc_area entries have been restored and all the in use chunks that have been dirtied are also restored. Although in principles this could entail an indefinite number of iterative backward steps along the log chain, in practice the restore operation can be immediately finalized once we find a full-log while backward re-traversing the log chain. In fact, all the in use chunks that have not yet been restored are immediately available inside the full log for copy-back operations. Actually, to optimize the detection of already restored chunks, which must therefore not be copied-back again from the log, the iterative restore procedure has been based on temporary bitmaps (each associated with an active malloc_area) on which a couple of fast bitwise XOROR operations are executed each time a dirty bitmap (associated with that same malloc_area) is extracted from the incremental log.

\subsubsection{Caching Write References for Latency Reduc- tion while Managing the Memory Map}

The data structures characterizing Di-NONAME-LIB, are based on the avoidance of per-chunk headers. This is an explicit design choice aimed at minimizing the amount of meta-data to be logged/restored $\left({ }^{2}\right)$. Hence, when a chunk gets released by the overlying application, no header information can be exploited for fast access to the malloc_area involved in the deallocation operation. To speedup deallocations, via the avoidance of a scan operation over all the active malloc_area entries, the original NONAME-LIB provided a software level direct-map caching subsystem, implemented as a hash table, with cache line formed by the couple < chunk_addr, m_area_index $>$.

The issue of identifying the correct malloc_area starting from the memory address associated with a chunk becomes even more critical in the new version DiNOINAME-LIB. Specifically, the memory map manager needs to retrieve the malloc_area for updating the information about dirty data/meta-data each time it receives an input from the update_tracker. This occurs each time an instrumented memory write operation dirtying whichever chunk inside the memory map occurs. Such an operation is likely to be more frequent compared to a deallocation operation. Also, in Di-NONAME-LIB we need to retrieve the correct malloc_area starting from a memory address which does not necessarily coincide with the chunk boundary address (as instead occurs for free operations). In other words, update_tracker could catch a write operation related to a memory location placed in the middle of a single chunk.

To cope with such an issue, the original cache has been extended by having the cache line augmented with the chunk end address and represented by the tuple < chunk_start_addr, chunk_end_addr, m_area_index >. Hence the cache as been actually modified to achieve a cache for multi-set inputs. The start address for a memory write operation intercepted by the update_tracker is stripped of $n$ less significant bits by the memory map manager and is then used as the key for accessing the hash table, the value of $n$ being chosen with the aim at making the whole range of addresses belonging to each single chunk collide into a single cache line. Actually, given that the size of the chunks delivered to the application software can be different $\left({ }^{3}\right)$, $n$ has been set as the mean value between the number of bits needed to make the smallest and the greatest chunks managed by Di-NONAME-LIB collide, biased to the smaller sizes.

\subsection{Interaction with Third Party Libraries}

With the original NONAME-LIB, any memory write operation on allocated chunks was allowed to occur inside

\footnotetext{
${ }^{2}$ Flexibility in memory management via partitioning/aggregating free memory buffers according to the so called "boundary tagging" scheme [2] is anyway inherited by Di-NONAME-LIB thanks to per-chunk headers used at the level of the underlying malloc library.

${ }^{3}$ We recall that, similarly to the malloc library, Di-NONAME-LIB manages different power of 2 chunk sizes, with a parameterizable maximum size typically set to $32 \mathrm{~KB}$.
} 
functions in third party libraries, provided that these functions did not allocate any further memory buffer (as is the case for most functions inside the $\mathrm{C}$ standard library stdlib). This is no longer automatically the case when using DiNONAME-LIB and its incremental log/restore facilities. In fact, libraries are not instrumented hence it would not be possible for update_tracker to catch memory changes made inside those libraries.

We have explicitly addressed the case of update operations performed by third party software, just focusing on stdlib. Specifically, we have implemented inside DiNONAME-LIB a set of function wrappers for all those functions whose signature allows the overlying software to pass a pointer for a memory write operation to be performed by the library. Those wrappers simply throw back the call to the underlying standard-library function, and then pass control to the memory map manager with explicit indication of the address of the updated buffer, and the size of the updated memory block. In case the size cannot be retrieved by the library function signature (as for pointers to buffers used for strings), the memory map manager is provided with a special flag, which triggers the manager to update the dirty bits for all the currently allocated contiguous chunks starting from the pointed address. This is obviously a conservative way of managing the memory map which can only result in an increased $\log /$ restore overhead (due to the fact that some chunks that have not been really dirtied by the library are actually considered as dirty ones). Correctness is in no way touched given that the wrapped library functions are all stateless, thus posing no issue on the side of memory $\log /$ restore.

Anyway, we are currently working on techniques for application transparent management, and integration with DiNONAME-LIB, of all those library functions which explicitly allocate memory and/or have an internal state.

\section{Related Work}

In the optimistic simulation context, several solutions have been introduced for logging the whole state of a simulation object (at each event execution or after an interval of executed events) $[10,15,17,18,20]$, or incrementally logging modified state portions [7, 21, 25, 27], or supporting a mix of the two approaches $[11,24]$. With these solutions there is the need (i) to supply the necessary code to collect snapshots of the objects' state inside the application level software, or (ii) to employ calls to functions within the API of proper checkpointing libraries, or (iii) to statically identify (e.g. at compile-time) which portions of the address space need to be considered part of the state. Consequently, perfect transparency is not supported since the programmer must necessarily be faced with issues related to state snapshots. Also, static identification of the memory locations to be included inside the snapshot is non-compatible with dynamic memory allocation/deallocation (e.g. via standard libraries) at the simulation object level. This is the case for the work in [27], which, as sketched in the introduction, has some technical similarities to our work on the side of automatic instrumentation, but does not allow dynamic memory to be employed. Compared to all those approaches, our solution supports state management, based on incremental $\log$ capabilities, without the need for specific log/restore modules within the application code, or for explicit interfacing with $\log$ /restore libraries, and allows the simulation object state to be scattered on dynamically allocated memory chunks.

The issue of dynamic memory based states for optimistic simulation objects has also been addressed by the optimistic simulation frameworks in [3, 9]. However, ad-hoc APIs are used to explicitly notify to the simulation kernel that specific allocation/deallocation operations, and, more in general, operations on data structures based on dynamic memory (e.g. lists), need to be rollbackable. Hence, differently from our approach, dynamic memory based layouts via ANSI-C memory allocation/deallocation services are not supported.

In terms of capabilities of the memory management subsystem the closest works to our approach are probably the ones in $[22,23]$, which present software layers for transparent log/restore in optimistic simulation based on the HighLevel-Architecture (HLA) interoperability standard. These layers rely on Operating System memory protection mechanisms to detect memory updates and to incrementally log dirty pages belonging to a dynamically changing federates' memory layout. Compared to our proposal, the overhead for tracking updates and incremental log operations is likely higher and affordable only when comparable with the cost of interoperability services supported by HLA middleware. This makes those approaches not tailored to traditional optimistic simulation platforms, which are instead the target of the present work.

Some recent advances $[5,8,14]$ have shown the viability and effectiveness of optimistic state management via reverse computation, where a reverse version of application level simulation code is (automatically, or semiautomatically) generated and employed for backward computation just aimed at restoring the state of the simulation object. Anyway, in general simulation contexts (e.g., possibly exhibiting non-reversible execution paths), this approach still needs to be complemented via optimized log/restore techniques like the one we have presented in this work.

Finally, our proposal is also related to a number of works in the field of program execution tracing (see, e.g., $[1,4,16,28])$ for debugging, vulnerability assessment and repeatability purposes. Compared to our work, several of those approaches provide detailed analysis of changes in the state of the program, and of the execution flow. However, this is achieved via performance intrusive techniques relying on dynamic instrumentation and/or kernel level services, unsuited in contexts (e.g. parallel simulation) where performance cannot be sacrificed. Again concerning program debugging supports, the only works showing basic operating mode comparable to our one (namely, the employment of trap mechanisms based on code insertion/replacement to detect memory write accesses) are those addressing data watch points (see, e.g., [26]. However they have performance targets different from ours since optimizations mostly cope with search techniques for verifying whether a memory reference falls inside a region that is currently subject to a watch point. In other words, aspects related to the identification of areas that have been dirtied and 
to incremental $\log /$ restore operations are not considered.

\section{Experimental Data}

We have integrated Di-NONAME-LIB within the open source NONAME optimistic simulator [19]. This is a traditional-style (event-handler based) optimistic simulation platform, which transparently supports all the mechanisms associated with parallelization (e.g. the mapping of simulation objects on different kernel instances) and optimistic processing. It relies on MPI for data exchange across different simulation kernel instances.

The hardware platform used in this experimental study is a Quad-Core machine equipped with four 2.4-GHz/4MBcache 64-bits Intel processors and 4 GB of RAM memory, running LINUX (kernel version 2.6.22). Each core hosts one instance of the optimistic simulation kernel.

The test-bed application software is a parameterizable cellular system simulator, explicitly modeling fading and channel interference phenomena [12]. Each simulation object instance models a single cell, by tracking, via dynamically allocated data structures, channel allocation and power management information for ongoing calls. Specifically, upon the start of a call destined to a mobile device currently hosted by the cell, the simulation object allocates a new call-setup record via a couple of dynamically allocated data structures, and links it to a list of already active records, each one released when the corresponding call ends or is handed-off towards a different cell (in this case a similar call-setup procedure is executed at the destination cell). Upon call-setup, power regulation is performed, which involves scanning the aforementioned list of records for computing the minimum transmission power allowing the currently setup call to achieve the threshold-level SIR value, according to GSM technology. Data structures keeping track of fading coefficients are also updated while scanning the list.

We have simulated macro-cells, each one managing up to 1000 wireless channels, using classical settings such as exponential distribution of the call inter-arrival time, and average call duration of 2 minutes (see, e.g., [6]). Also, the call inter-arrival frequency to each cell has been varied in the interval between 1 and 6.25 calls per simulation time unit, thus providing increasing values of the channel utilization factor (in between 12\% and 75\%), and hence increasing values of the expected length of the aforementioned list of in-use records. This has a twofold effect: (1) The storage requirement for the state of each simulation object varies in between $4 \mathrm{~KB}$ and $32 \mathrm{~KB}$ (meta-data for the maintenance of the memory map being excluded). (2) The event granularity grows from finer to coarser values. These variations allow us to evaluate the effects of the innovative capabilities provided by Di-NONAME-LIB in differentiated configurations.

For instrumented and non-instrumented software we comparatively report in Figure 3 the measured values for the below parameters, with measures obtained for a small-sized test-bed benchmark configuration formed by four simulation objects (each one hosted by one instance of the simulation kernel running on the Quad-Core machine):
(A) The average latency for the execution of a simulation event.

(B) The average latency for a log operation.

(C) The average latency for restoring the memory map to a logged state.

(D) The average size for a taken log.

As pointed out in Section 3.2.2, the latency of a state restore operation in Di-NONAME-LIB directly depends on the interleaving between full logs and incremental logs along the log chain. Hence, for the parameters in points (B), (C) and (D), the plots refer to different interleaving steps between full and incremental logs, namely full logs taken every 20 and every $50 \log$ operations, respectively. Also, non-instrumented software has been linked to the original version of NONAME-LIB in order not to alter the inner (optimized) logic and data-structures specifically designed for non-incremental logging.

By the results, we see that the overhead caused by the memory update tracking mechanism on the event execution latency is very limited. Also, CPU and memory requirements for each log operation in the instrumented case are definitely lower than those observed for non-instrumented software. The latter configuration actually provides a gain for state restore operations. Anyway, by the plots we see that performance decrease in the state restore for the case of instrumented software can be controlled (while maintaining the advantages on the side of logging) via proper selection of a non-oversized interleaving step between full and incremental logs.

In order to assess the overall benefits provided by $\mathrm{Di}$ NONAME-LIB, in Figure 4 we also report plots related to the event rate (committed events per wall-clock time unit) while varying the state log interval, also known as checkpoint period. This time the curves refer to a much larger configuration of the aforementioned benchmark, with 1024 macro-cells evenly distributed on the four simulation kernel instances hosted by the Quad-Core machine.

Concerning the checkpoint period, namely the independent parameter, we recall that it directly affects the tradeoff between state log and coasting forward overheads, and potentially affects the overall memory locality due to variations in the memory usage for checkpoints. For DiNONAME-LIB, a state restore (occurring before coasting forward, if any) additionally depends on the interleaving step between full logs and incremental logs, which has been set to 20 in this study. Also, the plots are reported for three different channel utilization factors $(25 \%, 50 \%$ and $75 \%)$ in order to observe the overall performance while varying the application software CPU/memory requirements.

By the results we see that, as soon as the application exhibits non-minimal memory requirements (namely when the channel utilization factor is non-minimal, hence inducing an increase in the simulation object state size), the nonincremental approach provides significant performance advantages. We note that these advantages come from a direct reduction in the cost of state logs (this can be noted especially for small values of the checkpoint period) and from 
increased locality (this can be noted especially for increased values of the checkpoint period, where the event rate curve for the incremental case does not stay flat, as instead occurs for the non-incremental case). Concerning the latter point, the frequency of GVT calculation and related memory recovery operations has been set in a way to never exceed $60 / 70 \%$ of RAM usage, so not to incur swapping phenomena that would alter the reliability of the reported measures. Hence, improved locality even excludes potential (further) advantages from incremental logging thanks to the avoidance of swap phenomena, which are more likely to occur with non-incremental logs in case of (excessively) lazy settings for GVT operations.

\section{Summary}

In this paper we have presented a software architecture complementing an existing open source layer supporting transparent $\log$ /restore operations for optimistic simulation objects with state layout based on standard dynamic memory allocation/deallocation services. The new capabilities added through the presented architecture entail: (i) Lightweight run-time monitoring mechanisms (based on ad-hoc software instrumentation facilities) for tracking memory update references inside the current memory map associated with the state of each simulation object - (ii) Optimized log/restore based on incremental copies of dirty chunks inside the memory map. Some experimental results have also been reported for an evaluation of the benefits achievable through the provided approach. Planned future work encompasses: (A) Supports for completely transparent state management, based on incremental logging, in the context of application software integration with third party libraries (for which we have currently provided a partial solution coping with stateless libraries, and libraries not directly interacting with lower level memory allocation/deallocaton APIs) - (B) The design of (autonomic) mechanisms for dynamic switching between incremental and non-incremental operating modes, in order to further improve the system run-time behavior by optimizing the trade-off between the cost of memory update tracking (to be paid in case the incremental mode is switched on) and the cost of (full or incremental) log operations.

\section{References}

[1] GDB: The GNU Project Debugger. http://www.gnu.org/software/gdb/

[2] A memory allocator. http://g.oswego.edu/dl/html/malloc.html, 1996.

[3] SPEEDES. http://www.speedes.com, 2005.

[4] V. Bala, E. Duesterwald, and S. Banerjia. Dynamo: a transparent dynamic optimization system. In Proceedings of the 2000 ACM SIGPLAN Conference on Programming Language Design and Implementation (PLDI), pages 1-12, 2000

[5] D. W. Bauer and E. H. Page. An approach for incorporating rollback through perfectly reversible computation in a stream simulator. In 21 st International Workshop on Principles of Advanced and Distributed Simulation, pages 171178. IEEE Computer Society, 2007.

[6] A. Boukerche, S. K. Das, A. Fabbri, and O. Yildz. Exploiting model independence for parallel PCS network simulation. In Proceedings of the 13th Workshop on Parallel and Distributed Simulation, pages 166-173. IEEE Computer Society, May 1999

[7] D. Bruce. The treatment of state in optimistic systems. In Proceedings of the 9th Workshop on Parallel and Distributed Simulation, pages 40-49. IEEE Computer Society, June 1995
[8] C. D. Carothers, K. S. Perumalla, and R. Fujimoto. Efficient optimistic parallel simulations using reverse computation. ACM Transactions on Modeling and Computer Simulation, 9(3):224-253, 1999.

[9] S. Das, R. Fujimoto, K. Panesar, D. Allison, and M. Hybinette. Gtw: a time warp system for shared memory multiprocessors. In WSC '94: Proceedings of the 26th conference on Winter simulation, pages 1332-1339, San Diego, CA USA, 1994. Society for Computer Simulation International.

[10] J. Fleischmann and P. Wilsey. Comparative analysis of periodic state saving techniques in Time Warp simulators. In Proceedings of the 9th Workshop on Parallel and Distributed Simulation, pages 50-58. IEEE Computer Society, June 1995.

[11] S. Franks, F. Gomes, B. Unger, and J. Cleary;. State saving for interactive optimistic simulation. In Proceedings of the 11th Workshop on Parallel and Distributed Simulation, pages 72-79. IEEE Computer Society, June 1997.

[12] S. Kandukuri and S. Boyd. Optimal power control in interference-limited fading wireless channels with outage-probability specifications. IEEE Transactions on Wireless Communications, 1(1):46-55, 2002.

[13] D. E. Martin, T. J. McBrayer, and P. A. Wilsey. WARPED: A time war simulation kernel for analysis and application development. In HICSS ' 96 : Proceedings of the 29th Hawaii International Conference on System Sciences (HICSS'96) Volume 1: Software Technology and Architecture, page 383, Washington, DC, USA, 1996. IEEE Computer Society.

[14] A. Naborskyy and R. M. Fujimoto. Using reversible computation techniques in a parallel optimistic simulation of a multi-processor computing system. In $21 \mathrm{st}$ International Workshop on Principles of Advanced and Distributed Simulation, pages 179-188. IEEE Computer Society, 2007.

[15] B. R. Preiss, W. M. Loucks, and D. MacIntyre. Effects of the checkpoint interval on time and space in Time Warp. ACM Transactions on Modeling and Computer Simulation, 4(3):223-253, July 1994.

[16] F. Qin, C. Wang, Z. Li, H.-S. Kim, Y. Zhou, and Y. Wu. Lift: A low-overhead practical information flow tracking system for detecting security attacks. In MICRO, pages 135-148, 2006

[17] F. Quaglia. A cost model for selecting checkpoint positions in Time Warp parallel simulation. IEEE Transactions on Parallel and Distributed Systems, 12(4):346-362, Feb. 2001.

[18] F. Quaglia and A. Santoro. Non-blocking checkpointing for optimistic parallel simulation: Description and an implementation. IEEE Transactions on Paralle and Distributed Systems, 14(6):593-610, 2003.

[19] Reference removed for blind review.

[20] R. Ronngren and R. Ayani. Adaptive checkpointing in Time Warp. In Proc. of the 8th Workshop on Parallel and Distributed Simulation, pages 110-117. Society for Computer Simulation, July 1994.

[21] R. Ronngren, M. Liljenstam, R. Ayani, and J. Montagnat. Transparent incremental state saving in Time Warp parallel discrete event simulation. In Proceedings of the 10th Workshop on Parallel and Distributed Simulation, pages 70-77. IEEE Computer Society, May 1996.

[22] A. Santoro and F. Quaglia. Transparent state management for optimistic synchronization in the High Level Architecture. In Proceedings of the 19th Workshop on Principles of Advanced and Distributed Simulation, pages 171-180 IEEE Computer Society, 2005.

[23] A. Santoro and F. Quaglia. A version of MASM portable across different UNIX systems and different hardware architectures. In Proceedings of the 9th International Symposium on Distributed Simulation and Real Time Applications. IEEE Computer Society, 2005.

[24] H. Soliman and A. Elmaghraby. An analytical model for hybrid checkpointing in Time Warp distributed simulation. IEEE Transactions on Parallel and Distributed Systems, 9(10):947-951, 1998.

[25] J. Steinman. Incremental state saving in SPEEDES using c plus plus. In Proceedings of the Winter Simulation Conference, pages 687-696. Society for Computer Simulation, 1993.

[26] R. Wahbe, S. Lucco, and S. L. Graham. Practical data breakpoints: Design and implementation. In Proceedings of the 1993 ACM SIGPLAN Conference on Programming Language Design and Implementation (PLDI), pages 1-12, 1993.

[27] D. West and K. Panesar. Automatic incremental state saving. In Proceedings of the 10th Workshop on Parallel and Distributed Simulation, pages 78-85. IEEE Computer Society, May 1996.

[28] Q. Zhao, R. M. Rabbah, S. P. Amarasinghe, L. Rudolph, and W.-F. Wong. How to do a million watchpoints: Efficient debugging using dynamic instrumentation. In L. J. Hendren, editor, CC, volume 4959 of Lecture Notes in Computer Science, pages 147-162. Springer, 2008. 

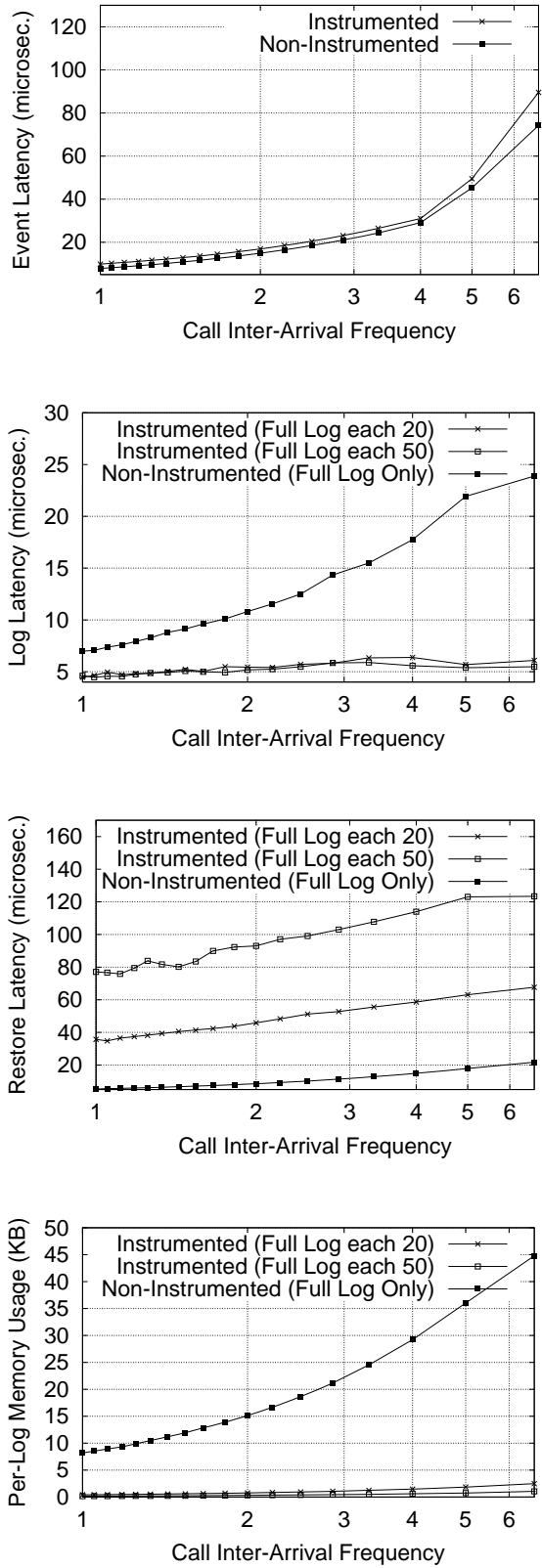

Figure 3. Basic Statistics for the Test-bed Configuration.
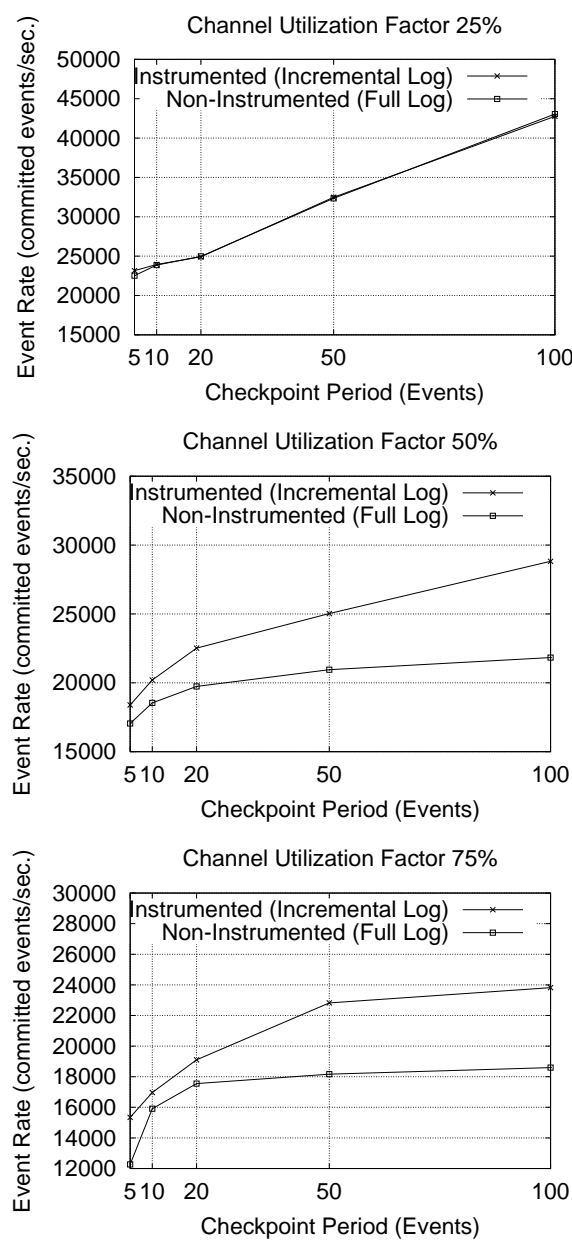

Figure 4. Event Rate vs the Checkpoint Period for Three Different Channel Utilization Factor Values (1024 Simulation Objects). 